\title{
Weiteres über Urgesteinsflora auf Flysch, Kreide, Lias und Trias.
}

\author{
Von Dr. J. Murr (Feldkirch).
}

In zwei früher (Allg. bot. Zeitschr., 1914, S. 133 ff., 1915, S. 25 ff.) erschienenen Artikeln habe ich aus den Florenverhältnissen Vorarlbergs und Liechtensteins dargelegt, wie infolge des sehr hohen Silikatgehaltes zunächst die oberste Schichte der älteren Kreide, der Gault, weiter der Flyschsandstein, aus der Trias der Buntsandstein, sowie (gleich dem Lias) infolge der tonigen Beimengung die Kössener Schichten urgesteinliebende Arten in mehr weniger starkem Prozentsatz beherbergen.

Um diesen Verbältnissen weiter nachzugehen, unternahm ich in den letzten Sommern trotz mancher Ersehwerung und Behinderung durch die Kriegsläufte eine Reihe von Ausflügen in den genannten geologischen Gebieten Vorarlbergs, welche meine früheren Ergebnisse in wünschenswerter Weise bestätigten und ergänzten.

So beging ich am 18. August 1915 neuerdings den Flyschsandstein des Bödele-Hochälple (1100-1460 m) bei Dornbirn ${ }^{1}$ ).

Die hier fast ausschließlich herrschende "Urgesteinsflor $\mathbf{a}^{\mathbf{2}}{ }^{2}$ ) ist dort in der Bergregion auf fenchtem, moorigem Waldboden durch Aspidium montanum, Blechnum spicant, Equisetum telmateia, E. silvaticum, Carex pendula, Stellaria uliginosa, Alchemilla pratensis, A. filicaulis, Chaerophyllum Villarsii, Circaea alpina, C. intermedia, Vaccinium myrtillus, Veronica montana, auf Heideboden durch Pteridium aquilinum, Deschampsia flexuosa, Sieglingia decumbens, Carex leporina, Stellaria graminea, Potentilla erecta, Calluna, Veronica officinalis, Gnaphalium dioicum, Solidago virga aurea, massenhafte

1) Einzelnes darüber bersits i. d. Allg. bot. Zeitschr., 1914, S. 133 und 1915, S. 25. Fast gleich wie der Flyschsandstein verhält sich die Molasse, auf der z. B. am Pfänder bei Bregenz fast ausschlieblich Urgebirgstypen, wie Pteridium, Lycopodium clavatum, Sieglingia, Alchemilla hybrida, A. crinita, Calluna, Campanula barbata, massenhafte Arnica, Hieracium laevigatum, H. vulgatum ssp. perscissum usw. auftreten.

2) Vollkommen kieselstete Arten gibt es ja wenige; doch läßt sich auch bei den Arten, welche sowohl auf Urgestein wie auf Kalk auftreten, fast in allen Fällen auf Grund der Erfahrung ein Hinneigen zur einen oder anderen Unterlage festsetzen; abgesehen von dieser selbstgewonnenen Einsicht halte ich mich an die bald strengere, bald weitere Fassung, wie ich sie in Werken und Einzelabhandlungen von Engler, Hegi, Vollmann, Dalla Torre u. Sarnthein, Handel-Mazzetti u.a. angewendet finde. 
Arnica und ebenso zahlreiches und formenreiches Hieracium vulgatum vertreten. Für das Voralpengebiet sind Lycopodium alpinum, Nardus stricta, Trichophorum austriacum, Carex brunnescens, C. magellanica, Luzula luzulina, Juncus filiformis, Veratrum album, Gymnadenia albida, Polygonum bistorta, Alchemilla crinita, Potentilla aurea, Rhododendron ferrugineum (ausschließlich), Vaccinium uliginosum, Gentiana Kochiana, Euphrasia minima, Campanula barbata, Gnaphalium silvaticum var. Einseleanum, Willemetia stipitata, Leontodon pyrenaicus, Hypochaeris uniflora, Hieracium vulgatum grex irrigunm, H. juranum ssp. prenanthopsis und integrifolium ssp. subvulsum charakteristisch.

Ähnlich gestalten sich die Verhältnisse uber Flyschsandstein auf den Bergwiesen bei Frastanz-Gurtis an der Südlehne des Illtales $(700-1100 \mathrm{~m})$. Von silikatliebenden Pflanzen treffen wir hier: Lycopodium clavatum, Botrychium lunaria, Deschampsia flexuosa, Poa nemoralis var. firmula (diese ganz ebenso auf Flyschsandsteinblöcken am Hohen Gerach), Nardus stricta, Carex pulicuris, Veratrum album, Orchis maculata, Gymnadenia albida, Alnus riridis, Thesium pratense, Polygonum viviparum, Potentilla aurea, Geum montanum (selten), Alchemilla pubescens, A. flabellata (war für Vorarlberg zweifelhaft), A. pastoralis, A. connivens, Hypericum maculatum, Silaus flevescens, Chaerophyllum Villarsii, Chamaenerion angustifolium, Calluna vulgaris, Vaccinium uliginosum, Gentiana Intea, G. Kochiana, Ajuga pyramidalis, Campanula barbata, Arnica montana (massenhaft), Solidago alpestris, Antennaria dioica, Scorzonera humilis, Hypochaeris uniflora, Hieracium pilosella ssp. subcaulescens, H. vulgatum ssp. aurulentum, ssp. perscissum, ssp. lonchodes, H. levigatum.

Bemerkenswert ist, wie unter fast gleichen Verhältnissen auf Unterlage von Flyschsandstein in Fellengatter-Amerlügen bei Feldkirch (600-750 m), entlang der Auslaugungen der mit tuffbewohnendem Eucladium verticillatum, Didymodon tophaceus, D. giganteus und Orthothecium rufescens ausgepolsterten oder eingefaßten Kalkbäehlein, eine echte Kalkflora aus Carex sempervirens, Gymnadenia odoratissima, Dryas octopetala, Erica carnea, Pinguicula alpina, Globularia mudicaulis, Valeriana saxatilis usw. entgegentritt.

Von silikatliebenden Laubmoosen beobachtete ich auf Filysehsandstein folgende Arten: Antitrichia curtipendula (Fellengatter, Gurtis), Barbula vinealis var. cylindrica (Fellengatter-Bodenwald), Bartramia lateralis (Fallenberg-Bödele bei Dornbirn), Blindia acuta (ebenda), Dicranodontium longirostre (ebenda), Dicranum longifolium (Fellengatter, Gurtis), Ditrichum homomallum (Bödele-Langkopf), Dryptodon Hartmanni (Fellengatter), Hedwigia albicans (ebenda), Hylocomium loreum 
(Bödele), Plagiothecium undulatum (ebenda), Pterigynandrum filiforme und var. ambiguum (Fellengatter), Rhacomitrium canescens (Gurtis), Rh. heterostichum (Fellengatter, Letze), Seligeria recurvata (Bödele).

Von Flechten sind Cetraria islandisa und Cladonia rangiferina auf Unterlage von Flyschsandstein allgemein zu finden, auf dem freiliegenden Flyschfels gedeihen die silikatliebenden Arten: Parmelia conspersa* (Letze, Fellengatter, Gurtis), Caloplaca flavovirens* (Letze), Aspicilia cinerea (Fellengatter, Gallmist), Variolaria lactea* (ebenda), Rhizocarpon obscuratum* (Gallmist), Rh. geographicum (Ludescherberg am Frassen, Hoher Gerach ${ }^{1}$ ). Dazu kommen die mir nachträglich von dem am 2. September d. J. auf einer lichenologischen Exkursion an der Heimspitze in Montavon abgestürzten Pilzforscher Prof. P. Ferd. The i $§$ en bestımmten Arten: Cladonia pungens* (Fellengatter), Cl. rangiformis* (Gurtis), Lecidea Pilati* (Ludescherberg).

Fast ebenso reich an silikatliebenden Arten wie der Fyschsandstein ist der durch seine massenhaften Chondrites-Einschlüsse so leicht kenntliche Flyschmergel, dem besonders die nordseitigen Hünge des Illtales von Satteins bis zum Hohen Frassen in ihrer unteren and mittleren Zone angehören. Schon durch das stellenweise Hervortreten der Pterideta und Nardeta, weiter oben dureh Alnus viridis, Populus tremula, Calluna, Vaccinium uliginosum, massenhafte Arnica und Campanulc barbata gibt sich der lebmige Flyschmergel sofort zu erkennen.

Daneben treten z. B. am Schnifnerberg bei $1400 \mathrm{~m}$ (fast gleich auch am unmittelbar anschließenden Dünserberg), gemischt mit kalkliebenden Arten, wie Sorbus chamaemespilus, Rosa pendulina, Daphne mezereum, Laserpitium latifolium, Crepis blatturioides, auch die anderen, schon früher genannten kiesel-oder lehmliebenden Typen entgegen, wie: Lycopodium clavatum, Deschampsia flexuosa, Carexpilulifera, C. pallescens, Luzula sudetica, Thesium pratense, Anemone narcissiflora, Alchemilla pubescens, Geum montanum (dieses selten), Vicia silvatica, Chaerophyllum Villarsii, Gentiana Kochiana, Euphrasia minima, Plantago alpina, Succisa pratensis, Phyteuma betonicifolium, Antennaria dioica, Gnaphalium norvegicum, Centaurea pseudophrygia, Scorzonera humilis var. angustifolia, Hypochaeris uniflora, Crepis conyzifolia (massenhaft), Hieracium vulgatum, bes. grex irriguum, H. juranum ssp. hemiplecum (approx.). Am Satteinserberg (900-1100 m) begegnet in ähnlicher Gesellschaft neben Laserpitium prutenicum und Silaus flavescens auch schon Gymnadenia albida; auch auf dem Flyschmergel des Vorder-

i) Die Flechten wurden, abgesehen von den gewöhnlichsten Arten, gröbtenteils durch Freund H. Zschacke in Bernburg bestimmt; die mit * bezeichneten Arten sind füz Vorarlberg neu. Die nicht gewöhnlichen Laubmoose sind durchgehends von Redakteur L. Löske revidiert. 
älple bei Feldkirch $(900-1250 \mathrm{~m})$ tritt neben viel Arnica, Centaurea pseudophrygia und zahlreichen Formen der Hieracia Vulgata, wie Hieracium vulgatum ssp. lonchodes und $H$. divisum ssp. vorarlbergense gleichfalls Laserpitium prutenicum zahlreich auf, das sich in Innsbruck nur als Relikt auf Schiefer, in Südtirol speziell auf Porphyr in der Kastanienzone, in Südsteiermark stellenweise als Charakterpflanze auf Mergel findet.

Am Dünserberg traf ich bei $1450 \mathrm{~m}$ sehr sparsam auch Agrostis rupestris, am Hohen Frassen (18. August 1917) bei $1500 \mathrm{~m}$ Calamagrostis tenella und Hieracium juranum in der ssp. prenanthopsis und angenähert der ssp. hemiplecum. Im Mazonenwald ober Laterns (5. Juli 1915) sammelte ich bei $1200 \mathrm{~m}$ Listera cordata, Carex brunnescens, Gnaphalium norvegicum, etwas höher auch Hieracium juranum.

An moorigen Stellen des Schnifnerberges bei $1500 \mathrm{~m}$ treten Epilobium alpinum und Juncus filiformis auf.

Die silikatliebenden Laubmoose treten auf Flyschmergel immerhin der Artenzahl nach sparsamer auf als auf dem Flyschsandstein. Ich fand am Dünserberg Campylopus fragilis, Cynodontium fallax, Dicranella subulata, Dicranodontium longirostre, Ditrichum homomallum, Mnium spinosum, Pterigynandrum filiforme, Rhacomitrium canescens und Webera clongata, am Frassen Polytrichum alpinum, ober Laterns gegen die Gaphal-Alpe massenhaft Plagiothecium undulatum.

Von Flechten beobachtete ich auf Flyschmergel bei Raggal im Großen Walsertale (6. Mai 1915): Diploschistes scruposus*, Variolaria lactea und Lecidea platycarpa*.

Noch weniger zahlreiche silikatliebende Arten weist der am Nordhange des Illtales über $1500 \mathrm{~m}$ und öfter wohl auch schon beträchtlich tiefer auftretende Flyschkalk auf. So bietet die von mir offters besuchte, ïber den Schnifnerberg gegen den Hohen Gerach hinaufziehende Geröllmulde eine uppige, fast reine Kalkflora mit Cystopteris regia, C. montana, Carex ferruginea, Moehringia muscosa, Ranunculus alpestris, R. montanus, Alchemilla pallens, Hedysarum obscurum. Imperatoria ostruthium, Heracleum montanum, Pedicularis foliosa, Doronicum grandiflorum, Cirsium spinosissimum, Salix arbuscula, S. hastata var. vegeta usw., nur daß auch hier die für die Flora von Vorarlberg so charakteristischen tonliebenden Arten, wie Anemone narcissiflora, Trifolium Thalii, Astragalus frigidus, Vicia silvatica, Plantago alpina, P. montana, Campanula thyrsoidea, Centaurea montana stärker hervortreten; höchstens Alchemilla decumbens könnte als silikatliebende Spezies aufgefuhrt werden. Erst an dem stärker verwitterten Querkamm, links unter dem Gerach, 
bei $1800 \mathrm{~m}$ fand ieh Gentiana nivalis und Agrostis rupestris. Die gegen den Hohen Gerach ziehende Mulde über der Hinteren Alpila zeigt vorwiegend Kalkflora, an Felsblöcken Sedum atratum, Alchemilla pallens, Hippocrepis, Helianthemum grandiflorum, Erica, Satureia alpina, Veronica aphylla, Globularia cordifolia, Galium anisophyllum, Seabiosa lucida, Carduus viridis; an feuchten Stellen aber finden sich neben Alnus viridis auch Carex frigida, Trifolium badium, Willemetia stipitata, vollends auf tieferem, moorigem Humus, der nach Auflösung und Fortsehwemmung des Kalkes übrig geblieben ist, kommt die schon des öfteren vorgeführte silikatiiebende Gesellsehaft mit Blechnum, Nephrodium montanum, üppigem Anthoxanthum odoratum, Stellaria graminea, Polygala vulgare, Euphrasia minima, Phyteuma betonicifolium zur Entwicklung. Noch ausgesprochener fand ich diese dem moorigen Humus angepaßte Vegetation bei $2000 \mathrm{~m}$ am Alpilakopf über der Hinteren Alpila-Alpe (15. Juli 1918), wo unter Rhododendron ferrugineum und Rh. intermedium zahlreicher Leontodon pyrenaicus, ja sogar Hieracium nigrescens ssp. pseudohalleri und spärliches Lycopodium alpinum gedeiben; von Pinus montana sah ich bezeichnenderweise im Umkreis dieses Gipfels nur ein Stück. Weiter links, an der Hauptspitze des Gerach (auf der Generalstabskarte „Kubspitze“, bestiegen am 22. August 1918), konnte ich von silikatliebenden alpinen Phanerogamen lediglich Rhododendron ferrugineum und Rh. intermedium sowio spärliches Hieracium aurantiacum notieren; Rom pel fand dortselbst Sedum annuum, St. Ka i s e r Oxyria digyna. Von silikatliebenden Laubmoosen fand ich auf Flyschkalk lediglich Rhacomitrium canescens, Dicrunum longifolium, Dicranoweisia crispula (diese in der vorderen Alpila-Mulde) und Hylocomium pyrenaicum (am Gerach-Gipfel), von Flechten außer Cetraria islandica auch Rhizocarpon geographicum.

An den Flyschkalk schließt sich der in seinen Eigensehaften und in seinem Verhalten recht ähnliche Vertreter der jüngeren Kreide, der Seewenkalk, an. Wo er massig und ausschließlich auftritt, wie z. B. am Nob $(1790 \mathrm{~m})$, einem Vorberge des Freschen (von mir am 5. Juli 1915 besucht), zeigt er an den freien, größtenteils des Humus entkleideten Hängen (gegen Norden und Nordwesten) die reme Kalkflora mit S'alix hastata var. vegeta, S. arbuscula, Rosa pendulina, spärlichem Rhododendron hirsutum, Carex ferruginea, Oxytropis montana, Hedysarum, Crepis montana, Hieracium villosum, H. chlorifolium ssp. pulchriforme usw.; die verflachte Kuppe mit ihrem geschlossenen, tieferen Humus hingegen bietet wieder größtenteils die bekannten silikatliebenden Typen, von denen Alnus viridis und Rhododendron ferrugineum, dann Trichophorum austriacum, Allium sibiricum, Geum montanum, Alchemilla crinita, Euphrasia minima, Scorzonera humilis var. angustifolia, 
Willemetia, Leontodon pyrenaicus, Hieracium alpinum und $H$. integrifolium ssp. subvulsum besonders genannt sein mögen.

Am Freschen und auf Gamperney der Alviergruppe (St. Gallen) tritt der Seewenkalk infolge ursprünglicher Faltung in Bändern, mit breiteren Gaultschichten abwechselnd, anf und hier konnte auch manche silikatliebende Art von dem ihr freilich weit bessere Bedingungen bietenden Gault auf die Seewenschichten abbersiedeln. Ich beobachtete auf Seewenkalk am Freschen Avenastrum versicolor, Luzula spicata, Trifolium pallescens, Gentiana bavarica, Euphrasia minima, auf Gamperney (16. September 1918) gletchfalls Avenastrum versicolor und Luzula spicata, dann Phododendron intermellium, Cerastium lanatum und Alchemilla alpina ssp. alpina. Auf der benachbarten Alpe Schlawitz bei Grabs (von mir besucht am 26. August 1910 und 10. Juli 1914) liegt der Seewenkalk von etwa $1400 \mathrm{~m}$ an in geschlossener Masse über dem Gault. Hier beobachtete ich von silikatliebenden Arten Rhododendron ferrugineum, Lloydia serotina, Cerastium strictum, Silene rupestris, Sedum annuum, Saxifragu moschata, Hieracium nigrescens ssp. nigrescentiforme und H. cydoniifolium. Das sonst Kallflora bietende Trümmer- und Geröllfeld an der Alpe Palfries (besucht an 24. Juli 1908, 3. und 8. Angust 1911 und 1. August 1912) enthält merkwürdigerweise Hieracium piliferum wie verschiedene Zwischentormen der Hieracia Prenanthoidea, z. B. H. juranum, H. macilentum ssp. macilentiforme, $I$. cydoniffolium ssp. Cottianum, H. Sulgeri mh.

An silikatliebenden Moosen finden wir auf Seewenkalk, doch auch nur spärlich und zerstreut, zum guten Teil dieselben weniger anspruchsvollen Arten, die uns schon da und dort anf Flysch begegneten, wie Barbula vinealis var. cylindrica (Letze b. Feldkirch), Bartramia lateralis (ober Emsreute), Brachythecium reflexicum (Freschen), Bryum Duvalii (Schlawitz), Dichodontium pellucidum (ebenda), Dicranoweisia crispula (Freschen, Schlawitz), Eurhynchium cirrhosum (Schlawitz), Rhacomitrium cancscens (Freschen, Schlawitz), Webera elongata (ob Emsreute).

Durch sehr hohen Kieselgehalt (bis zu 85\%) stellt sich dem Flysehsandstein der Gault, die oberste Schichte der älteren Kreide, zur Seite, welche in der unteren Region, zumal um Feldkirch, fast als Charaktergestein mit seinem stufenartigen Bruche in schwarzbrauner, melaphyrähnlicher Färbung und von gelblichen Eisenausscheidungen durchzogen, allerwärts (wie am Ardetzenberge, Blasenberge und Schellenberge, im Stein- und Göfnerwalde, an der Rückseite des Stadtschrofens, in der Duxgasse bei Tisis) über den mächtigen Bänken des Urgon zutage tritt. Intolge seines xerothermen Charakters ist das Vorhandensein dieses 
Gesteines auch bei geschlossener Humusdecke an dem Vorwiegen der Föhre mit Pteridium, Calluna (neben der aber bezeichnenderweise öfter, wie z. B. am "Stein" bei Feldkirch und am Freschen, anch Erica auftritt), Vaccinium myrtillus, Populus tremula und Ilex als Unterwuchs sofort zu erkennen.

Von krautartigen Gewächsen finden wir auf diesem heideartigen Waldboden sehr zahlreiche silikatliebende Typen, wie Polypodium vulyare, Lycopodium clavatum, Deschumpsia caespitosa, D. flexuosa, Anthoxanthum odoratum, Holcus lanatus, Sieglingia decumbens, Carex leporina. C. pilulifera, C. pallescens, Luzula multiflora, Rumex acetosella. Stellaria graminea, Potentilla erecta, Alchemilla pubescens. A. pastoralis (diese bei Feldkirch nur am Ardetzenberge von mir gefunden), A. acutangula, Hypericum humifusum (Stadtschrofen), Polygala vulgare, Veronica officinalis, Antennaria dioica, Solidago virga aurea, Hypochaeris radicata, Hieracium auricula, H. sabandum.

Eine ebenso charakteristische silikatliebende Gesellschaft findet sich auf Gault im humusreicheren, feuchteren Hochwalde, der um Feldkirch, besonders um Gö fis, schon bei $600 \mathrm{~m}$ fast typisch ausgeprägt ist. Es sind dies Arten wie Polypodium vulgare, Nephrodium oreopteris, Blechnum spicant, Equisetum silvaticum, Lycopodiun annotinum. L. selago, L. complanatum, Deschampsia caespitosa, Carex pulicaris, C. echinata. Juncus conglomeratus, Luzula luzulina, Orchis maculata, Stellaria uliginosa, Alchemilla alpestris, A. filicaulis, A. pratensis. Rubus idaeus, Hypericum maculatım, Chamaenerium angustifolium, Chaerophyllum Villarsii, Vaccinium myrtillus, V. vitis idaea, Digitalis ambigua, Veronica montuna, Prenanthus purpurea, Willemetic stipitata.

Von silikatliebenden südlichen Relikten möchte ich aus der Felsenflora des Gault im besonderen noch Asplenium adiantum nigrum (sehr spärlich am Ardetzenberg, bei Tosters und im Göfnerwald), Rumex scutatus (Wasserschloß am Ardetzenberg) und Potentilla argentea (Blasenberg und Ardetzenberg) anführeu.

Zur Vorfübrung der silikatliebenden Vertreter der alpinen Gaultflora möge die (mit Bändern von Seewenkalk durchsetzte) Gipfelregion des Freschen $(1700-2000 \mathrm{~m})$ dienen, den ich am 13. September 1915 und 5. September 1918 bestieg. Ich nenne hier: Nardus stricta, Anthoxunthum odoratum, Deschampsia flexuosa, Avenastrum versicolor, Agrostis rupestris, Trichophorum austriacum, Carex pallescens, C. leporina, Luzula spadicea, L. spicata (beide sebr spärlich), L. sudetica, Gymnadenia albida, Polygonum viviparum, Cerasticum trigynım. Sempervivum alpinum, Alchemilla glaberrima, Geum montanum, 
Potentilla erecta, P. aurea, Trifolium pallescens, T. badium, Hypericum maculatum, Polygala vulgare, Chaerophyllum Villarsii, Epilobium alpinum, Soldanella pusilla, Calluna vulgaris, Rhododendron ferrugineum, Vaccinium vitis idaea, V. myrtillus, V. uliginosum, Gentiana bavarica, G. Kochiana, Euphrasia minima, Campanula barbata, C. Scheuchzeri, Arnica montana, Solidago alpestris, Gnaphalium norvegicum, G. supinum (spärlich), Leontodon pyrenaicus, Hypochaeris uniflora, Hieracium valdepilosum ssp. valdepilosum, $H$. alpinum und ssp. Halleri ${ }^{1}$ ).

Zur Ergänzung mögen zunächst noch Artenverzeichnisse von mehr weniger benachbarten Vorbergen und Voralpen, die gleichfalls dem Gault angehören, vorgeführt werden, nämlich vom $\mathrm{Kulm}$ bei Übersaxen, $900-1100 \mathrm{~m}$, von der Voralpe Furx, $1170 \mathrm{~m}$, und ron Schutanna-Hinterberg bei Hohenems, $1100-1450 \mathrm{~m}$ (letztere Voralpen besucht am 2. und 23. August 1916). Ich traf an diesen Orten: Nephrodium montanum, Blechnum spicant (Hinterberg), Equisetum silvaticum, Lycopodium clavatum (Kulm), Sieglingia decumbens (Schutanna), Agrostis rupestris (Hinterberg), Nardus stricta (Furx, Schutanna), Luzula luzulina (Kulm, Furx), Coeloglossum viride (Furx), Listera cordata (Hinterberg), Gymnadenia albida (Kulm), Polygonum viviparum (Kulm, Furx), P. bistorta (Schutanna), Alchemilla pubescens, A. crinita (Furx), Hypericum humifusum (Schutanna), Laserpitium prutenicum, Silaus flavescens (Kulm), Vaccinium uliginosum, Gentiana Kochiana (Kulm, Furx), Euphrasia minima. Campanula barbata, Arnica montana, Antennaria dioica (Furx), Centaurea pseudophrygia, Crepis grandiflora (Kulm), Hieracium stoloniflorum, H. juranum ssp. prenanthopsis, ssp. elegantissimum, H. integrifolium ssp. subvulsum (Hinterberg).

In besonders reicher Zahl treten die silikatliebenden Typen in der Gaultzone des benachbarten schweizerischen Alvierstockes auf, wo ich die Alpen A rin (25. und 28. Juli 1907, 11. Juli 1908, 22. Juli 1910, 20. Juli 1914) und Gamperney (16. September 1918) näher untersuchte. Ich nenne von hier, unter Weglassung gewöhnlicher, schon vom Freschen genannter Typen: Polypodium vulgare (G.), Avenastrum versicolor (A.), Agrostis rupestris (A., G.), Carex brunnescens, C. magellanica (A.), Luzula spadicea (A., G.), Juncus filiformis, Silene rupestris, Cerastium lanatum, C. strictum, Sedum dasyphyllum (G.), S. annuum, S. alpestre (A.), Alchemilla alpina ssp. alpina! (A., G.), A. colorata,

1) Carex curvula (Custer) vom Freschen halte ich für bedenklich, außerdem werden noch Cerastium lanatum (Custer), Sedum annuum (Rompel), Saxifraga bryoides (Rehsteiner), Empetrum nigrum (A. Sauter) und Saussurea alpina (Custer) vom Freschen angegeben. 
A. connivens (G.), A. glaberrima, Geum montanum, Sibbaldia procumbens, sehr spärlich (A.), Potentilla sabauda, Ligusticum simplex (G.), Epilobium alpinum, Soldanella pusilla (A.), Calluna vulgaris, Rhododendron ferrugineum ausschließlich, Vaccinium vitis idaea, V. uliginosum (A., G.), Gentiana nivalis, Veronica bellidioides, Phyteuma betonicifolium (G.), Ph. hemisphaericum, Gnaphalium norvegicum (A., G.), G. supinum, spärlich (G.), Adenostyles alliariae, Chrysanthemum alpinum ssp. hutchinsiifolium (A.), Leontodon pyrenaicus (A., G.) Hieracium fuscum ssp. fuscum (A.), H. alpinum und ssp. Halleri (G.), H. nigrescens ssp. nigrescentiforme (A.. G.), H. vulgatum ssp. lonchodes (G.), H.juranum ssp. prenanthopsis, ssp. pseudojuranum, ssp. elegantissimum, ssp. juranum, H. integrifolium ssp. subalpinum, H. Beauverdianum, H. picrioides ssp. picrioides (A.) ${ }^{1}$ ).

Trotz allem überwiegt, abgesehen von bodenvagen und von lehmliebenden Arten, wie Anemone narcissiflora, Trifolium Thalii, Astragalus frigidus, Vicia silvatıca, Plantago montana, P. alpina, Chrysanthemum atratum, Achillea macrophylla auf den freien, weniger humosen Stellen des Gault die Kalkflora. So treffen wir auf dem Gipfel des Freschen, zum Teile in hervortretender Weise, Arten wie Phleum Michelii, Gypsophila repens, Sedum atratum, Rosa pendulina, Hedysarum obscurum, Anthyllis alpestris, Daphne striata, Atamantha cretensis, Erica carnea, Primula farinosa, P. auricula, Androsace chamaejasme, Myosotis alpestris var. pseudosuaveolens mb., Satureia alpina, Veronica fruticans, Euphrasia salisburgensis, Globularia cordifolia, Galium helveticum, Scabiosa lucida, Campanula cochlearifolia, Aster bellidiastrum, A. alpinus, Erigeron polymorphus, Doronicum calcareum, Carduus viridis, Leontodon taraxaci, Hieracium villosum. Doch habe ich ziemlich zahlreiche der entschieden auf Kalk angewiesenen Arten wie Carex firma, Moehringia ciliata, Arabis bellidifolia, A. pumila, Draba aizoides, Hutchinsia alpina, Thlaspi rotundifolium, Kernera saxatilis, Biscutella levigata, Saxifraga caesia, S. stellaris, Valeriana saxatilis, Leontodon incanus, Hieracium bupleuroides, am Freschen und sonst in der alpinen Zone auf Gault nicht beobachtet.

Ziemlich stark treten auch unter den Laubmoosen auf Gault die silikatliebenden Arten hervor. Es sind dies für die untere Zone (zum Teil als Glazialrelikte) Arten, wie Antitrichia curtipendula (Göfnerfeld), Barbula botelligera (Duxgasse bei Tisis, sehr selten), Barbula

1) Über die Gaultflora der Alpe Arin habe ich bereits in der Allg. bot. Zeitschrift, 1914, S. 135 ff., berichtet, sehe mich aber veranlabt, diese Angaben im Zusammenhalte mit den so gut entsprechenden und ergänzenden über die Alpe Gamperney, den Freschen usw. zu wiederholen. 
vinealis var. cylindrica (Ardetzenberg Norden, Carina), Bryum Mildeanum (Tosters, Ardetzenberg, Hofen, Göfnerfeld), Campylopus flexuosus (Göfnerfeld, Stadtschrofen), C. fragilis (ebenda), Dichodontium pellucidum (Duxgasse), Dicranodontium longirostre (Göfnerwald), Ditrichum pallidum (Ardetzenberg, Stadtschrofen), Dryptodon Hartmanni (Ardetzenberg, Norden), Grimmia commutata (Duxgasse), G. ovata (Göfnerfeld, doch wohl von erratischem Gneis übergesiedelt), Hedwigia albicans (Ardetzenberg, Duxgasse, Carina; sehr spärlich und dürftig entwickelt), Mniobryum albicans (Margarethenkopf, Steinwald, Altenstadt), Philonotis caespitosa (Göfnerwald), Plagiothecium Ruthei (Amberger Wald), Pleuridium subulatum (Ardetzenberg, Stadtschrofen), Pogonatum urnigerum (Ardetzenberg, Steinwald nsw.), P. nanum (Göfnerwald), Polytrichum piliferum (Steinwald, selten), Pterygophyllum lucens (Ardetzenberg Norden, Amberger Wald), Rhacomitrium heterostichum (Duxgasse), Rh. canescens (Ardetzenberg, Hofen, Steinwald, St. Arbogast), Seligeria recurvata (Ardetzenberg, Duxgasse, Grabs), Tortula rurulis (Ardetzenberg usw.)

Fast noch zahlreicher finden sich die silikatliebenden Laubmoosarten auf Gault in der alpinen Zone. Ich nenne: Amplidium Mougcotii (Arin), Bartramia lateralis (ob Emsreute, Gamperney, Grabs), B. ithyphylla (Arin, Gamperney), Brachythecium reflexum (Arin). Bryum Inuvalii (Schlawitz), Br. pallescen. (Gamperney), Camplopus fragilis (Freschen), Coscinodon cribrosus (Hohe Kugel; es handelt sich hier wie bei den anderen von der Hohen Kugel angeführten Moosen und den Flechten nur um einen vereinzelten, auf der Kammhöhe liegenden Gaultblock), Dichodontium pellucidum (Freschen, Schlawitz), Dicranella squarrosa (Schutanna-Hinterberg), D. subulata (ebenda und Arin), Dicranoweisia crispula (Muttkopf, Furx, Freschen, Hohe Kugel, Arin), Dicranum Starkei (Freschen, Arin), D. albicans (Gamperney), Dryptodon Hurtmanni (Furx, Arin, Gamperney), Eucalypta ciliata (Gamperney), Hedvigia albicans (Schlawitz), Heterocladium squarrosulum (Arin), IHylocomium loreum (Hinterberg). H. pyrenaicum (Arin), Hypnum callichroum (Arin), Oligotrichum hercynicum (Freschen), Philonotis fontana acced. ad tomentellam (Freschen), Playiothecium undulatum (Schutanna-Hinterberg), Pogonatum urnigerum (Muttkopf), Pohlia commutata (Freschen), Polytrichum alpinum (Freschen, Arin, Gamperney), $P$. juniperinum (Freseben, Gamperney), P. piliferum (Gamperney), Pterigynandrum filiforme (Schutanna, Arin, Gamperney), Rhacomitrium canescens var. ericoides (Muttkopf, Freschen, Hohe Kugel, Hinterberg, Arin), Rh. fasciculare (Arin), Rh. heterostichum (Arin), Seligeria recurvata (Gamperney), Sphagnum compactum (Freschen), Webcra. acuminata (Arin), W. elongatu (ob Emsreute, Hinterberg). 
An silikatliebenden Lebermoosen nenne ich von Arin: Jungermannic confertifolia, J. barbata, Nardia geoscyphus, Scapania helvetica.

Dasselbe Verhältnis wie bei den Moosen herrseht auch bezüglich der Flechtenflora des Gault. Von silikatliebenden stellte ich im Gebiete folgende Arten fest:

Parmeliella microphylla* (Duxgasse), Parmelia conspersa (Veitskapf am Ardetzenberg, Göfnerfeld), Caloplaca flavovirens (Veitskapf, Tisis), Placodium murale (Veitskapf, Carina-Tisis, Hohe Kugel), P. circinatum* (Veitskapf. Tisis), Lecanora subfusca var. campestris (Tisis), L. polytropa (Furx), Aspicilia gibbosa* (Veitskapf, Tisis, Hohe Kugel), A. cinerea (Tisis, Duxgasse), Diploschistes scruposus (Veitskapf, Tisis, Göfnerfeld), Variolaria lactea (Göfnerfeld), Biatora coarctata* (Veitskapf, Tisis, Göfnerwald), Lecidea confluens* (Furx), L. platycarpa* (Duxgasse), L. enteroleuca (Veitskapf, Duxgasse, Tisis, Göfnerwald, Furx), L. latypaea (Hohe Kugel), Catocarpus badioater: (ebenda), Rhizocarpon geographicum (Hohe Kugel, Freschen), R. concentricum* (Duxgasse, Göfnerfeld), ferner die vier mir heuer von P. Ferd. Th $\in$ i $ß$ en bestimmten Arten: Aspicilia caesiocinerea* (Hohe Kugel), Buellia epipolia* (Tisis), Lecidea crustulata* (Hohe Kugel), Physcia lithotea* (Veitskapf). Selbstverständlich kommen anf Gault überall im Gebirge auch die silikatliebenden Astflechten Cetraria islandica und Cladonia rangiferina vor (auf Gamperney vergesellschaftet mit Cetraria islandica var. crispa, C. juniperina und Alectoria ochroleuca).

Die mittlere Schichte der jüngeren Kreide, der Urgon (Schrattenkalk) kommt im Aussehen und Verhalten der Trias am nächsten und bietet im allgemeinen die reine Kalkflora sogar dort, wo die unmittelbare Nähe des Gaultsandsteines ein Übertreten silikatliebender Arten erleichtern würde. Öfter, wie z. B. im Gebiete der Hohen Kugel und des Freschen ist er, offenbar infolge ungleichmäßiger Auftragung (oder, nach einem Volksausdruck, "Auslassens" der Schichte), fast gänzlich unterdrückt (auf der Hohen Kugel liegen sogar, infolge gleichzeitigen Fehlens des Gault, das Neokom and der Seewenkalk unmittelbar nebeneinander), und nur einzelne herumliegende Stücke yon Urgon geben uns Kenntnis von dessen Vorhandensein. An solchen kleinen Trümmern des Urgon tritt bei der Verwitterung der reiche Gehalt an feinen Glimmerteilchen klar zutage, so daß solche Stücke äußerlich einem Flyschsandstein nahekommen und so auch ausgesprochen kieselliebenden Flechten eine Lagerstätte bieten, wie ich denn auf der Hohen Kugel an solchen verwitterten Urgontrümmern Lecidea crustulata* und L. latipaea, mein damaliger (6. August 1917) Begleiter Lehrer K. Zerlauth sogar Rhizocarpon geographicum sammelte. 
Die im Gebiete oft mächtig entwickelte unterste Schichte der älteren Kreide, das Neokom, kommt im Ansehen dem so lehmreichen Flyschmergel am nächsten. ohne aber im allgemeinen die gleiche Zahl silikatliebender Arten zu beherbergen. Im Gegenteile bietet z. B. die Mörzelspitze bei Dornbirn (bestiegen am 16. August 1918) mit ihren großartigen Schichtungen über der Alpe Unterfluh eine reine, blätenreiche Kalkftora mit Gypsophila, Cerinthe alpina, Campanula cochlearifolia, Leontodon hyoseroides, Crepis alyestris, C. blattarioides usw., höebstens daß zerstreutes Trifolium badium, Hypericum maculatum, Polygonum viviparum oder zahlreicheres Equisetum silvaticum den reschen Tongehalt des Bodens andeuten; erst der von geschlossener Grasnarbe bedeckte Gipfel $(1832 \mathrm{~m})$ bringt die bekanntesten silikatliebenden Alpinen, meist in ganz spärlicher Anzahl, wie Nardus, Agrostis rupestris, Geum montanum (1 Exemplar gefunden), Gentiana Kochiana, Euphrasia minima, Phyteuma betonicifolium, Leontodon pyrenaicus (1 Ex.), Hieracium furcatum, H. substoloniflorum (2 Ex.), H. alpinum ssp. melanocephalum ( $1 \mathrm{Ex}$.)

Besser steht die Sache bezüglich der silikatliebenden Arten im Bereiche der Hohen Kugel $(1650 \mathrm{~m})$ und des unmittelbar gegenüberliegenden First (gleichfalls $1650 \mathrm{~m}$ ), auf welchen zwei Gipfeln übrigens das Neokom in etwas bärterer Form auftritt. Schon über Fraxern geben ziemlich ausgedehnte Pterideta mit Laserpitium prutenicum, Serratula tinctoria ssp. austriaca und Centaurea pseudophrygia, dann wieder Populus tremula, Calluna, Sieglingia, Nordus, Gentiana Kochiana, Amica, Antennaria dioica den Mergelgehalt des Bodens zu erkennen. Am Gipfel der Hohen Kugel (zum ersten Male besucht am 12. Juli 1915, vollständiger begangen am 23. Juli 1917) fand ich neben vorwiegender Kalkflora Lycopodium alpinum, Agrostis rupestris, Carex magellanica (sehr spärlich), Alchemilla pubescens, A. colorata, A. flabellata, A. connivens, Gentiuna nivalis (ganz einzeln), Rhododendron ferrugineum. (vorwiegend), Euphrasia minima (neben Eu. hirtella), Campanula Scheucheri, Leontodon pyrenaicus, Hieracium stoloniflorum, H.Peterianum, auf dem First (9. August 1915 und 31. Juli 1916) Rhododendron ferrugineum, Blechnum, Trichophorum austriacum, Juncus filiformis, Gymnadenia albida, dürftigen Streptopus, Euphrasia minima, Gnaphalium norvegicum, Willemetia und Hieracium nigrescens ssp. pseudohalleri. Am Abstieg ron der Hohen Kugel zur Fluhereckalpe (6. August 1917 und 1. August 1918) kommt der Lehmgehalt des Neokom durch massenbafte Alnus viridis, Rhododendron ferrugineum (neben $R h$. hirsutum), Vaccinium uliginosum, Equisetum silvaticum, Blechnum, Nephrodium oreopteris, Anthoxanthum odoratum, Carex leporina, C. pallescens, Potentilla aurea, üppiges Trifolium badium, Campanula Scheuchzeri, 
Solidago alpestris, Achillea macrophylla, Willemetia, Hieracium pilosella ssp. subcaulescens, H. substoloniflorum, H. juranum ssp. prenanthopsis, H. epimedium zum Ausdruck. Auch an dem der Hohen Kuge! benachbarten Hörnle (31. Juli 1916) sammelte ich zwischen Alnus viridis, Rhododendron intermedium and Salix hastata var. vegeta (letztere kalkliebend), Hieracium juranum ssp. hemiplecum und $H$. integrifolium.

Auf dem lehmigen Neokomhange unterhalb der Voralpe Furs gegen Suldis herab treten in charakteristischer Häufigkeit Equisetum telmateia, Molinia arundinacea var. altissima, Vicia silvatica, Laserpitium prutenicum, Gentiana asclepiadea, Stachys officinalis, Knautic dipsacifolia z. T. etwas gegen $K$. drymeia neigend, Succisa pratensis var. Knautiifrons, Arnica montana, Serratula tinctoria ssp. austriaca entgegen, eine Pflanzengesellschaft, die sich genau gleich am Südostfuße der Alpen auf Mergelboden bei Marburg a. D. (250 m) findet.

An silikatliebenden Laubmoosen beobachtete ich auf Neokom nur Dichodontium pellucidum, Hylocomium loreum (Alpe Alpweg am Freschen ;. $_{\text {. }}$ H. pyrenaicum, Mnium spinosum (Hohe Kugel), Plagiothecium undulatım (gegen Alpe Unterfluh am Mörzel), Pterigynandrum filiforme (Hohe Kugel), Rhacomitrium canescens, Webera elongata (First).

Reich an silikatliebenden Arten ist der tonige Lias, dessen Flor: ich in ausgedehntem, reinem Bestande am 7. August 1916 am Hange östlich von der Ravensburger Hütte am Arlberg $(1900 \mathrm{~m})$ in Augenschein nahm.

Gebüsche von Alnus viridis und Rhododendron ferrugineum, im Grasteppich reichliche Deschampsia flexuosa, Avenastrum versicolor, Luzula multiflora, Gentiana purpurea, Hypochoeris uniflora, Hieracium fuscum ssp. chrysophanum, dazwischen vereinzelt Geum montanum, Alchemilla glaberrima, Euphrasia minima, an feuchten Stellen zahlreiche Carex frigida und Willemetia wie Gentiana bavarica bringen den Eindruck gleicher Hänge im Schiefergebirge mit sich. Auch auf die linke Bachseite herüber, unter der Ravensburger Hütte, setzt sich, völlig überwachsen, der Lias noch fort ${ }^{1}$ ); ich fand dort in ähnlicher wie der eben vorgeführten Gesellschaft auch je ein Individuum von Luzula spicata ssp. conglomerata und Hieracium alpinum ssp. Halleri. Am 6. August 1915 hatte ich bereits den Lias zwisehen Zürs und dem $\mathrm{Z} u ̈ r$ er See $(1800-2100 \mathrm{~m})$ begangen und dort zahlreiche urgesteinsliebende Arten notiert wie: Agrostis rupestris, Sibbaldia procumbens

1) Herr Kollege $K . G$ u n z hatte die Güte, hier wie an der Hohen Kugel die geologischen Verhältnisse nachzuprüfen, wobei sich völlige Übereinstimmung mit meinen Annahmen ergab. 
(sehr spärlich), Anthoxanthum odoratum, Juncus triglumis, Carex frigida, Allium sibiricum, Salix herbacea, Alchemilla glaberrima, Geum montanum, Calluna, Gentiana, Veronica bellidifolia, V. alpina, Phyteuma betonicifolium, Gnaphalium supinum, Chrysanthemum alpinum ssp. hutchinsiifolium, Leontodon pyrenaicus. Auf dem Lias der Roten W and am Lech-Ursprung $(2700 \mathrm{~m})$ wurden gefunden: Trisetum spicatum (J. Schwimmer), Lloydia serotina, Oxyria digyna (stud. Grill), Alsine recurva (Rehsteiner), Saxifraga bryoides, Geum reptans, Ligusticum simplex, Androsace alpina (Konr. Azwanger), Phyteuma pedemontanum, Artemisia Genipi, Saussurea alpina.

Wir kämen nun noch zur Trias, die ihrem Wesen nach silikatliebende Florenelemente im allgemeinen ausschließt. Doch auch hier gibt es mehr oder weniger lehmhältige Schichten, so zunächst die an den Lias unmittelbar anschließenden Kössener Schichten. Begreiflicherweise tritt auch vielfach (wie zwischen Gault und Seewenkalk) ein Austausch der Florenelemente dieser nächst benachbarten Schichten ein. So sammelte ich am 6. August 1915 auf Felspartien unterhalb des Zürser Sees am Arlberg: Avenastrum versicolor, Cerastium lanatum, Saxifraga moschata, Potentilla sabauda, Androsace obtusifolia, Grentiuna nivalis, Phyteuma hemisphaericum, Erigeron uniflorus, Hieracium piliferum, H. alpinum und ssp. Halleri.

Ganz ähnlich boten mir am 7. August 1916 die den gleichen Schichten angehörigen Felspartien hinter dem Spullersee: Agrostis rupestris, Sempervivum alpinum, Saxifraga moschata, Potentilla sabauda, Alchemilla pubescens, Vaccinium vitis idaea, Gentiana Kochiana, G. nivalis, Veronica alpina, Euphrasia minima, Erigeron uniflorus, Leontodon pyrenaicus. Vom Schafberg $(2680 \mathrm{~m})$ brachte mein Begleiter auf jener Partie, Koll. Jos. Rauch, Luzula spadicea, Cerastium lanatum, Geum montanum, Ligusticum simplex, Gentiana nivalis und Euphrasia minima mit.

Wie man sieht, sind es immer ganz bestimmte unter den silikatliebenden Arten, welche sich, wenn auch meist spärlich, auf den mehr oder weniger silikathältigen Schichten der Kreide oder der Trias zu halten vermögen.

Der Hauptdolomit zeigt natürlich im allgemeinen reine Kalkflora. Nur an Stellen mit tieferem, moorigem Humus kann sich auch hier eine silikatliebende Gesellschaft zusammenfinden, wie am linksseitigen Hange der Alpe Muttersberg (1400 m) am Hohen Frassen bei Bludenz (besucht am 20. Juli 1915), wo ich eine zumeist hochstengelige Vegetation mit Typen wie Calamagrostis villosa, Poa nemoralis, Aconitum napellus, Alchemilla pubescens, A. connivens, Chamaenerium 
angustifolium, Chaerophyllum Villarsii, Verbascum thapsus, Digitalis ambigua, Solidago virga aurea, Arnica montana, Crepis conyzifolia, Centaurea pseudophrygia, Hieracium aurantiacum, H. prenanthoides ssp. perfoliatum und ssp. strictissimum, $H$. juranum ssp. juranum, ssp. prenanthopsis und ssp. vipetinum ${ }^{1}$ ), also eine richtige Urgebirgsgesellschaft aus mittlerer Höhenzone. antraf. Ebenso fand ich im Walde unter Masescha (Triesenberg, Liechtenstein) auf einer Dolomitseholle viel Chaerophyllum Villarsii, Sambucus racemosa, Prenanthes purpurea und Hieracium juranum ssp. prenanthopsis.

In ihrem Verhalten den Kössener Schichten ähnlich zeigen sich die von mir am 1. September 1916 begangenen Raibler Schichten in Monteneu $(1800 \mathrm{~m})$ ob Schruns-Bartholomäberg, allwo jch, allerdings nur vereinzelt, mehrere stark silikatliebende Arten wie Rhododendron ferrugineum, Agrostis rupestris, Empetrum nigrum, Gentiana nivalis and Erigeron uniflorus, von Moosen Rhacomitrium canescens und Polytrichum alpium antraf.

Auf Arlbergkalk bei Stuben findet sich auker Chamaenerium Fleischeri und den von mir dortselbst zuerst für Vorarlberg beobachteten silikatliebenden Arten Rosa pomifera, Lathyrus heteroplayllus und Anthriscus nitidus besonders um Rauz eine reiche Fülle von Hieracia Prenanthoidea mit Arten und Unterarten wie Hieracium prenunthoides ssp. bupleurifolium and ssp. strictissimum, H. juranum ssp. prenanthopsis, $H$. epimedium ssp. macilentiforme, $H$. integrifolium ssp. subvulsum, H. inuloiles ssp. tridentatifolium ${ }^{2}$ ), von Piloselloidea: Hieracium aurantiacum und $H$. fuscum ssp. chrysophanum, endlich an einer Stelle auch zahlreiche Cropis conyzifolia.

Auf stark kristallinisch durchzogenem Arlbergkalk ob Gaflei (Liechtenstein) fand ich bei $1600 \mathrm{~m}$ am 18. August spärliche Narlus stricta und Vaccinium uliginosum sowie neben Euphrasia hirtella auch Eu. minima, auf Geröllstücken Placodium murale, Diploschistes scruposus, Lecidea lapicida* und Rhizocarpon geographicum.

Noch besser kommt die silikatliebende Gesellschaft auf dem hieher gehörigen Partnach-Mergel zur Entwicklung. Auf dieser Unterlage traf ich bei $1200 \mathrm{~m}$ an den Tußhütten in Liechtenstein (6. Juli 1918)

1) Die spärlichen Prenanthoidea-Standorte der weiteren Innsbrucker Umgebung finden sich ausschließlich auf Phyllit; nur im Herbare Zimmeter erliegt ain von diesem angeblich bei St. Magdalena im Halltale, also wohl auf Dolomit, gesammeltes H. juranum ssp. pseudohemiplecum.

2) Vgl. meine Übersicht „Die Hieracien des Arlberges" im 44. Jahresberichte des Museum-Vereines Bregenz über das Jahr 1906, S. 33-47. Von der Gruppe der Alpina sah ich hier trotz der unmittelbaren Nähe des Phyllits nie ein Stück auf den Kalk übergehen. 
einen Heideboden mit Juniperus, zwergiger Crataegus oxyacantha und Prunus spinosa, dann Calluna, Pteris, Anthoxanthum, Sieglingia, Antennaria dioica, bei $1500 \mathrm{~m}$ am Rellseck ob Bartholomäberg in Montafon (1. September 1916) Nardus stricta, Euphrasia minima, Leontodon pyrenaicus. Auf Muschelkalk am Hinter älple bei Feldkirch (30. Juli 1918) begegnen bei $1600 \mathrm{~m}$ Rhododendron ferrugineum, Vaccinium uliginosum, Alnus viridis, Nephrodium montanum, Equisetum silvaticum, Nardus stricta, Gnaphatium norvegicum und spärliche Alchemilla glaberrima. Dabei ist jedoch die Nähe einer stark lehmigen Schichte, in diesem Falle des Flyschmergels, in Betracht zu ziehen, von dem aus die Einwanderung auf die überschobene Trias stattfinden konnte.

Als unterste Schichte der Trias bleibt noch der Buntsandstein zu behandein, der in Aussehen und Verhalten lebhaft an den roten Quarzporphyr Südtirols erinnert. An dieser Schichte war mir zuerst bei einem Besuche des Triesenerberges (1100 m) am 30. Juli 1908 das Vorkommen ausgesprochen silikatliebender Arten und Formen wie Asplenium septentrionale, Poa nemoralis var. glausa, Silene rupestris und Sempervivum alpinum aufgefallen und am 22. Angust 1913 fand ich ebendort an Felstrümmern Dicranum longifolium, Grimmia ovata, G. decipiens, Dryptodon Hartmanni, Thacomitrium canescens, Ulota americana, Antitrichia curtipendula und Pterigynandrum filiforme.

Auf der oben erwähnten Strecke Schruns-Monteneu tritt ober Barthomäberg der Buntsandstein als ziemlich schmales Band gleich ober dem Phyllit entgegen. Hier traf ich in einer Waldschlucht bei $1200 \mathrm{~m}$ die nun schon hinlänglich oft vorgeführte, charakteristische, silikat- oder tonliebende Pflanzenfamilie, bestehend aus den Arten Alnus viridis, Sambucus racemosa, Pteridium aquilinum, Blechnum spicant, Nephrodium montanum, Calamagrostis villosa, Deschampsia flexuosa, Luzula nemorosa, Vaccinium myrtillus, Chaerophyllum Villarsii, Phyteuma betonicifolium, Hypochaeris radicata, Prenanthes purpurca, Hieracium juranum ssp. prenanthopsis, H. integrifolium ssp. subvulsum, H. silvaticum ssp. crepidiflorum.

Auch gegen St. Anton i. M. herab, also bei ca. $800 \mathrm{~m}$, macht sich der Buntsandstein durch das Auftreten von Pteridium, Polypodium vulgare, Blechnum, Anthoxanthum, Holcus lanatus, Deschampsia flexuosa, Luzula nemorosa, Chaerophyllum Villarsii, Vaccinium myrtillus, Digitalis ambigua und Prenanthes purpurea bemerkbar.

An silikatliebenden Moosen fand ich dort an einer humosen, feuchten Felswand von Buntsandstein Burtramia lateralis, Cynodontium strumiferum, Hedwigia und Webera elongata. 
Wenn wir einiges aus den Ergebnissen der oben dargelegten Nachforschungen zusammenfassen, so finden wir, was die jüngeren Schichten betrifft, im Gebiete nur auf Flysch und Kreide Lycopodium alpinum und Hieracium nigrescens (Empetrum nigrum auch auf Trias). speziell auf Flyseh und Gault Carex brunnescens, Sedum annuum und Hieracium picrioides, nur auf Flys ch Senecio carniolicus und Hieracium acratum ssp. Schroeterianum ${ }^{1}$ ), anf Flysch und Lias Oxyria digyna und Hypochoeris uniflora, auf Kreide (Seewenschichten und Gault) Cerastium lanatum, C. strictum, Silene rupestris (diese auch auf Buntsandstein), Alchemilla alpina ssp. alpina und Trifolium pallescens, auf Gault und Lias Saxifraga bryoides, Alchemilla glaberrima (diese von mir spärlich auch in der Trias gefunden), Sibbaldia procumbens, Ligusticum simplex, Soldanella pusilla, Veronica bellidioides (diese von Vollmann auch auf Flysch gefunden), Gnaphalium supinum, Chrysanthemum alpinum, dazu auf jüngerer Kreide und auf Lias Luzula spicata und Lloydia serotina, auf Gault und Kössener Schichten Luzula spadicea, Sempervivum alpinum, Phyteuma hemisphaericum, auf Seewenkalk und Kössener Schichten Hieracium piliferum, nur auf Lias Trisetum subspicatum, Alsine recurva, Saxifraga Seguierii, Geum reptans, Androsace alpina und Phyteuma pedemontanum. Auf den jüngeren Schichten weiter verbreitet sind Avenastrum versicolor, Saxifraga moschata, Geum montanum, Gentiana nivalis, Phyteuma betonicifolium, Erigeron uniflorus, Crepis conyzifolic und Hieracium alpinum.

Von 45 kieselliebenden alpinen Arten notierte ich 15 auf Flysch, 30 auf Gault (33 auf Kreide überhaupt), 20 auf Lias und 15 auf Kössener Schichten. Für den Flysch stellt sich das Verhältnis in Wirklichkeit bedeutend günstiger, da er in den mittleren und unteren Lagen stärker vertreten ist. Die Kössener Schichten nehmen, wie schon angedeutet wurde, wohl hauptsächlich deshalb eine bevorzugte Stellung in der Trias ein, weil sie dem Lias unmittelbar benachbart sind.

1) Letztere zwei Angaben wie noch einige wenige andere habe ich nach Dr. Vollmanns Aufsatz "Die Vegetationsverhältnisse der Allgäuer Alpen“ (vgl. meine Ausführungen i. d. Allg. bot. Zeitschr., 1915, S. 25) einbezogen; doch jst hier wie insbesondere leider auch bei vielen älteren Angaben aus dem Rhätikon die Schichte (Flysch, Kreide, Trias) nur im allgemeinen festgestellt, während ja doch, wie wir gesehen haben, die Unterabteilungen dieser Hauptschichten große Verschiedenheiten hinsichtlich des Silikat- oder Tongehaltes und demgemäß auch hinsichtlich des Vorkommens kieseliiebender Arten autweisen. 